\title{
Efecto del ejercicio agudo sobre la expresión del receptor tipo Toll-4 y los mecanismos inflamatorios en corazón de rata
}

\author{
CARLOS CRISTI-MONTERO ${ }^{1,2, a}$, PILAR SÁNCHEZ-COLLADO ${ }^{1, b}$, \\ CHRISTIANO VENEROSO ${ }^{1, \mathrm{c}}$, MARÍA JOSÉ CUEVAS ${ }^{1, \mathrm{~d}}$, \\ JAVIER GONZÁLEZ-GALLEGO ${ }^{1, \mathrm{e}}$
}

${ }^{1}$ Instituto de Biomedicina (IBIOMED) Universidad de León, España. Universidad Viña del Mar, Chile.

aprofesor de Educación Física, PhD.

${ }^{\text {b}}$ Farmacéutica, PhD. 'Profesor de Educación Física. PhD.

dBióloga. PhD.

eMédico y Biólogo. MD $\mathrm{PhD}$.

Recibido el 12 de octubre de 2011, aceptado el 5 de junio de 2012

Correspondencia a: Prof. Dr. Carlos Cristi Montero.

Universidad Viña del Mar. Chile. Agua Santa 7055, sector Rodelillo, Viña del Mar.

E-mail: uvmcristi@yahoo.es

\section{Effect of an acute exercise bout on Toll-like receptor 4 and inflammatory mechanisms in rat heart}

Background: Toll like receptor 4 (TLR-4) is a protein located in the cell membrane with an important function in the immune response of the organism. Its activation decreases heart contractility and activates nuclear transcription factor kappa B $(\mathrm{NF}-\kappa B)$. This in turn, increases the synthesis of different pro-inflammatory cytokines and the inducible enzyme nitric oxide (iNOS), which plays an important role in the inflammatory processes when nitric oxide production is enhanced. Aim: To determine if, after one session of acute exercise, expression of TLR-4 and iNOS, and activation of NF- $\kappa B$ are induced in rat cardiac tissue. Material and Methods: Exercise and control groups of eight male Wistar rats each, were studied. The exercise group was subjected to an acute exercise bout lasting one hour. After the exercise, the heart was excised to measure the expression of iNOS and TLR-4 genes by quantitative polymerase chain reaction, NF- $\mathrm{K} B$ activation by electrophoretic mobility shift assay (EMSA) and 550 by Western blot. Results: After exercise, there was an increase in TLR-4 and of $i N O S$ mRNA levels ( +46.7 and $+74.3 \%$ respectively). NF- $\kappa B$ activation and the nuclear expression of its p50 subunit also increased significantly ( +240 and $+306 \%$ respectively). Conclusions: Increased expression of TLR4 following a session of acute exercise may contribute to the activation of the NF- $\mathrm{K} B$ signaling route, promoting the synthesis of nitric oxide, which could influence negatively the cardiac response to high intensity physical exercise.

(Rev Med Chile 2012; 140: 1282-1288).

Key words: Excercise; Nitric oxide synthase Type II; Rat; Toll-Like receptor 4.
L as enfermedades cardiovasculares son la principal causa de muerte a nivel mundial ${ }^{1}$. También es ampliamente conocido el efecto cardioprotector, y las recomendaciones mínimas que debe tener un programa de ejercicio físico para lograr beneficios sobre diversas enfermedades $^{2}$, influyendo de esta manera en la disminución tanto de la morbilidad como de la mortalidad de la población ${ }^{3}$. No obstante, los efectos sobre la salud de una sesión aguda de ejercicio de alta intensidad no han sido tan extensamente estudiados.

La evidencia sugiere que el ejercicio prolongado e intenso causa una disfunción transitoria del miocardio, reduciendo la función tanto sistólica como diastólica ${ }^{4}$. De hecho, el ejercicio agudo se asocia con un incremento en los biomarcadores de daño miocárdico: troponinas cardiacas y pétido natriurético tipo $B$, tanto en atletas de élite como 
en sujetos que practican deporte recreacional ${ }^{5}$. Por tanto, realizar este tipo de ejercicio podría actuar como detonante en ciertos acontecimientos no deseados, como infarto agudo de miocardio y muerte súbita en individuos susceptibles ${ }^{6}$.

La razón por la que sujetos aparentemente sanos y sin signos de enfermedad miocárdica muestran concentraciones elevadas de estos biomarcadores no está totalmente establecida. No obstante, está demostrado que, incrementos en los contenidos de citoquinas proinflamatorias modulan fuertemente la función cardiaca y pueden inducir daño en los cardiomiocitos ${ }^{7}$.

Los receptores tipo Toll (TLRs) son proteínas de transmembrana que desempeñan un papel importante en la respuesta inmune del organismo ${ }^{8}$, estando también presumiblemente involucradas en diferentes aspectos de la respuesta inflamatoria mediante el reconocimiento de ligandos endógenos producidos durante la inflamación, incluso en ausencia de infección ${ }^{9}$. Uno de los TLRs mejor caracterizados, el TLR-4, se encuentra presente en células del sistema inmune pero también en adipocitos, hepatocitos y miocitos ${ }^{10}$. Su activación parece disminuir la contractilidad del corazón a través del factor nuclear kappaB $(\mathrm{NF}-\kappa \mathrm{B})^{11}$, tras la activación de sus subunidades p50 y p65 que, al translocarse al núcleo, inducen la síntesis de diversas citoquinas y enzimas proinflamatorias como la óxido nítrico sintasa inducible (iNOS) ${ }^{11}$. Esta enzima, al incrementar exageradamente la producción de óxido nítrico, juega un importante papel en la producción tanto de mediadores proinflamatorios, como en la apoptosis celular de cardiomiocitos ${ }^{12}$.

Hasta el momento no existe ninguna investigación sobre la expresión de TLR-4 en tejido cardiaco tras una sesión de ejercicio agudo. Por tanto, el objetivo del presente estudio fue examinar si existe un aumento en la expresión génica de ARNm del receptor TLR-4, la activación de NF- $\kappa B$ y la expresión de la actividad enzimática iNOS tras la realización de un protocolo de ejercicio físico agudo, en el corazón de ratas.

\section{Material y Métodos}

\section{Animales y procedimientos experimentales}

Los experimentos fueron realizados siguiendo los principios rectores para la investigación con animales ${ }^{13}$. Ratas macho raza Wistar (Charles
River, Barcelona) fueron alojadas en un ambiente controlado con ciclos diarios de $12 \mathrm{~h}$ de luz/oscuridad, temperatura ambiente de $22^{\circ} \mathrm{C}$ y comida (Panlab, Barcelona, España) y agua ad libitum.

Se dividieron en 2 grupos: 8 ratas en el grupo con ejercicio (peso corporal: $206 \pm 10 \mathrm{~g}$ y peso del corazón $0,73 \pm 0,04$ g) y 8 en el grupo control (peso corporal: $216 \pm 6 \mathrm{~g}$ y peso del corazón: 0,75 $\pm 0,04 \mathrm{~g})$.

\section{Protocolo de ejercicio}

El protocolo de ejercicio comenzó con un breve período de familiarización de $10 \mathrm{~min}$ a una velocidad de $8 \mathrm{~m} / \mathrm{min}$. Posteriormente, los animales fueron sometidos a una sesión aguda de ejercicio consistente en una carrera de una hora de duración, sobre un tapiz rodante (Letica, L18706; Letica, Barcelona, España) con 10\% de inclinación sobre una superficie horizontal y una velocidad constante de $25 \mathrm{~m} / \mathrm{min}^{14}$.

\section{Procedimiento quirúrgico y recogida de muestras}

Tras dos horas de reposo post-esfuerzo, se procedió a la disección quirúrgica de los animales para la obtención del corazón. Para ello fueron previamente anestesiados, vía intraperitoneal, con pentobarbital sódico $(50 \mathrm{mg} / \mathrm{kg}$ de peso corporal), manteniendo una temperatura aproximada de $37^{\circ} \mathrm{C}$ para evitar los efectos negativos de la hipotermia. La intervención comenzó con la disección de la arteria carótida izquierda, introduciendo en ella un catéter con anticoagulante y procediéndose a la exanguinación del animal como método de eutanasia. Posteriormente, se extrajo el corazón, que fue inmediatamente sumergido en nitrógeno líquido y almacenado a $-80^{\circ} \mathrm{C}$ para su posterior análisis.

\section{Retrotranscripción-Amplificación cuantitativa del ARN mediante PCR a tiempo real (RT-PCR)}

La expresión de ARNm de los genes iNOS y TLR-4 se cuantificó mediante RT-PCR ${ }^{15}$, utilizando el gen hipoxantina fosforibosil transferasa (HPRT) como control interno. El ARN total del músculo cardiaco fue aislado con el kit SV Total RNA Isolation System (Promega, Inc., Madison, WI) y cuantificado en un espectrofotómetro $\mathrm{Na}$ nodrop (ND-1000, Nanodrop, USA) ${ }^{16}$. El ADN genómico residual se eliminó tras incubación con DNasa-I libre de RNasas (Ambion Inc., USA). El ADN complementario (ADNc) se sintetizó a 
partir de 2 mg de ARN utilizando el Kit HighCapacity cDNA Archive (Applied Biosystems, Foster City, CA) y fue amplificado con TaqMan Universal PCR Master Mix (Applied Biosystems). Tanto las sondas y "primers" TaqMan específicos para TLR4 (acceso a Genbank no AF057025.1 y Rn00569848-m1), iNOS (acceso a Genbank no X76881.1 y Rn00561646-m1) y HPRT (acceso a Genbank no X62085.1 y Rn01527840-m1) se obtuvieron de TaqMan1 Assays on-Demand Gene, (Applied Biosystems). Los cambios relativos en los niveles de expresión génica se determinaron usando el método del $2^{-\triangle \Delta C T}$ como previamente se ha descrito ${ }^{17}$.

\section{Ensayo de Movilidad Electroforética (EMSA)}

Para estudiar la activación de $N F-\kappa B^{18}$ se obtuvieron extractos nucleares homogenizando $50 \mathrm{mg}$ de tejido cardiaco en $2 \mathrm{ml}$ de tampón A [10 mM Hepes $\mathrm{NaOH}$ (pH 7,9), 1,5 mM MgCl, $10 \mathrm{mM} \mathrm{KCl}, 1 \mathrm{mM}$ DTT, $1 \mathrm{mM}$ PMSF] con Nonidet $0,1 \%$. Tras 15-60 min de incubación en hielo, las muestras se centrifugaron $10 \mathrm{~min}$ a $1.000 \mathrm{xg} \mathrm{a} 4^{\circ} \mathrm{C}$, descartándose el sobrenadante. El pellet se resuspendió en $2 \mathrm{ml}$ de tampón A sin Nonidet, posteriormente se agitó vigorosamente y se centrifugó durante $10 \mathrm{~min}$ a $1.000 \mathrm{xg}$ a $4{ }^{\circ} \mathrm{C}$. Al pellet resultante se le añadieron $50 \mu \mathrm{l}$ de tampón B [20 mM Hepes- $\mathrm{NaOH}$ ( $\mathrm{pH} 7,9), 25 \%$ glicerol, $420 \mathrm{mM} \mathrm{NaCl}, 1,5 \mathrm{mM} \mathrm{MgCl}, 0,2 \mathrm{mM}$ EDTA (pH 8), 0,5 mM DTT, 0,5 mM PMSF]. Posteriormente, se incubó en hielo durante $30 \mathrm{~min}$ y se centrifugó a $4^{\circ} \mathrm{C}$ durante $15-30 \mathrm{~min}$ a $14.000 \mathrm{xg}$ para recoger el sobrenadante que se almacenó a $-80^{\circ} \mathrm{C}$ hasta su posterior análisis.

La concentración de proteínas se cuantificó mediante la técnica de Bradford ${ }^{19}$. Para la realización del EMSA se utilizó un método consistente en la separación electroforética del complejo NF- $\kappa \mathrm{B}$ y una sonda específica de ADN marcada radioactivamente. Para el marcaje de la sonda se utilizó un oligonucleótido de doble cadena ( $5^{\prime}$-AGT TGA GGG GAC TTT CCC AGG C-3') obtenido de Promega (Madison, USA) marcado con $\gamma^{-32} \mathrm{P}$ (ATP) y T4 PNK (polinucleotido kinasa) ${ }^{18}$. La sonda marcada fue almacenada a $-20^{\circ} \mathrm{C}$ durante un máximo de 15 días.

La reacción de unión al ADN fue realizada mediante la incubación de $25 \mu \mathrm{g}$ de extractos nucleares, $5 \mu \mathrm{l}$ de buffer $[10 \mathrm{mM}$ Tris/ $\mathrm{HCl}, 40$ $\mathrm{mM} \mathrm{NaCl}, 1 \mathrm{mM}$ EDTA y glicerol 4\%, pH 7,5], 1 $\mu \mathrm{g}$ Poli (dI-dC) y $\mathrm{H}_{2} \mathrm{O}$ estéril hasta completar un volumen total de $25 \mu$ l. Después de $15 \mathrm{~min}$ en hielo, $2 \mu \mathrm{l}$ del oligonucleótido marcado fue añadido a la mezcla, incubándose $20 \mathrm{~min}$ a temperatura ambiente. Con el fin de verificar que los resultados del EMSA no se deben a uniones inespecíficas, se llevaron a cabo experimentos de competición utilizando un control negativo o sonda fría, que contiene todos los reactivos excepto la muestra y una muestra de tejido cardiaco + un competidor no específico (sonda NC). Para la sonda NC se usó un oligonucleótido con una secuencia diferente a la secuencia citada anteriormente, en este caso el oligonucleótido SP1 (Promega Corporation, Madison, USA).

Las muestras se sometieron a electroforesis a través de un gel de poliacrilamida al $6 \%$ durante $90 \mathrm{~min}$ a $150 \mathrm{~V}$ a $4^{\circ} \mathrm{C}$. El gel se desecó al vacío $\left(80^{\circ} \mathrm{C}\right)$ y se introdujo en una casete hermética con una película de alta sensibilidad (Amersham Pharmacia, Piscataway, USA), incubándose a $-80^{\circ} \mathrm{C}$, 7 días. Tras el revelado de la película se realizó la cuantificación de las bandas en el densitómetro de imagen Scion Image (Maryland, USA).

\section{Western blot}

Para el análisis por Western blot ${ }^{20,21}$ de p50, en extractos nucleares, se utilizaron $75 \mu \mathrm{g}$ de proteína. Las proteínas fueron separadas por electroforesis en un gel de poliacrilamida (12\%) y posteriormente transferidas a una membrana de PVDF. Para bloquear la unión no específica, se preincubó la membrana durante $30 \mathrm{~min}$ a $37^{\circ} \mathrm{C}$ en una solución de bloqueo (PBS-Tween, $4 \%$ de leche desnatada). La membrana fue incubada toda la noche a $4^{\circ} \mathrm{C}$ con el anticuerpo específico para p50 (50 kDa) (Cell Signaling Technology, Beverly, USA). La detección de las proteínas se realizó por quimioluminiscencia (ECL; Amersham, Buckinghamshire, UK). Para visualizar las bandas proteicas en las membranas, estas fueron puestas en un casete con una película de rayos $\mathrm{X}$ (Hyperfilm ECL; Amersham Pharmacia, Uppsala, Sweden) y cuantificadas utilizando el Scion Image. La expresión proteica fue normalizada a través de la expresión de GAPDH (36 kDa) (Sigma, St. Louis, USA).

\section{Estadística}

La media y el error estándar de la misma (E.E.M) fueron calculados para todos los datos 


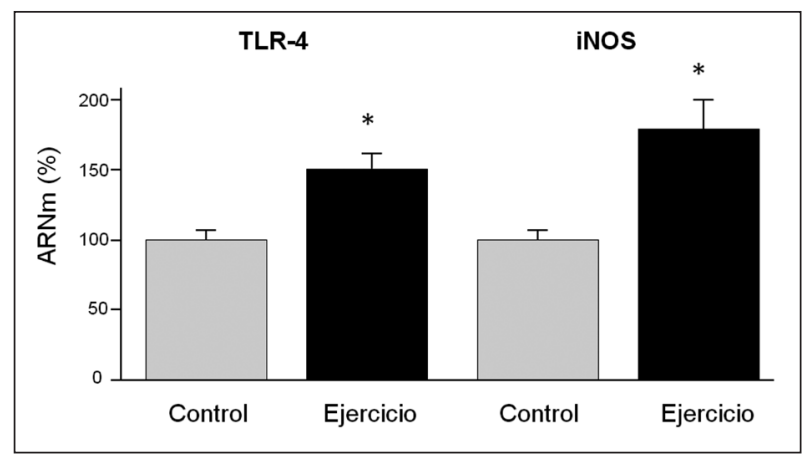

Figura 1. Contenidos de ARNm de TLR-4 e iNOS medidos a través de la técnica RT-PCR en tejido cardiaco de ratas sometidas a un protocolo de ejercicio físico agudo. Valores medios \pm E.E.M $(n=16) .{ }^{*} p=0,032($ TLR-4) y $p=0,017$ (iNOS) respecto a control.

del estudio. Para las comparaciones entre los grupos, se utilizó una prueba $t$ para grupos no pareados (MannWhitney test). Un valor de $\mathrm{p}<0,05$ fue considerado significativo. El tratamiento estadístico se realizó con el software GraphPad Prism (GraphPad Software, Inc., San Diego, CA) para MAC.

\section{Resultados}

Tras someter a las ratas al protocolo de ejercicio agudo, se observó que el ARNm de TLR-4 e iNOS, medido a través de la técnica RT-PCR, se incrementaba de manera significativa en $46,7 \%(\mathrm{p}=0,0032)$ y $74,3 \%$ $(\mathrm{p}=0,0017)$ respectivamente, al ser comparados con el grupo control (Figura 1).

Además, la activación de NF- $\kappa \mathrm{B}$, medida en extractos nucleares de tejido cardiaco a través de la técnica EMSA, aumentó en 195\% ( $\mathrm{p}=0,008)$ (Figura 2). También se observó un incremento significativo de 306\% $(\mathrm{p}=0,001)$ en la expresión de la subunidad p50 nuclear, medida por Western blot (Figura 3 ).

\section{Discusión}

El ejercicio físico está considerado como una herramienta complementaria al tratamiento de diversas afecciones cardiacas y como medio de prevención de las mismas. Esta protección podría estar relacionada con la ruta del TLR-4, ya que se ha demostrado que el ejercicio realizado de manera regular disminuye la activación de dicho receptor. Además, en ratones con déficit (inducido) de TLR-4 se ha demostrado una me-

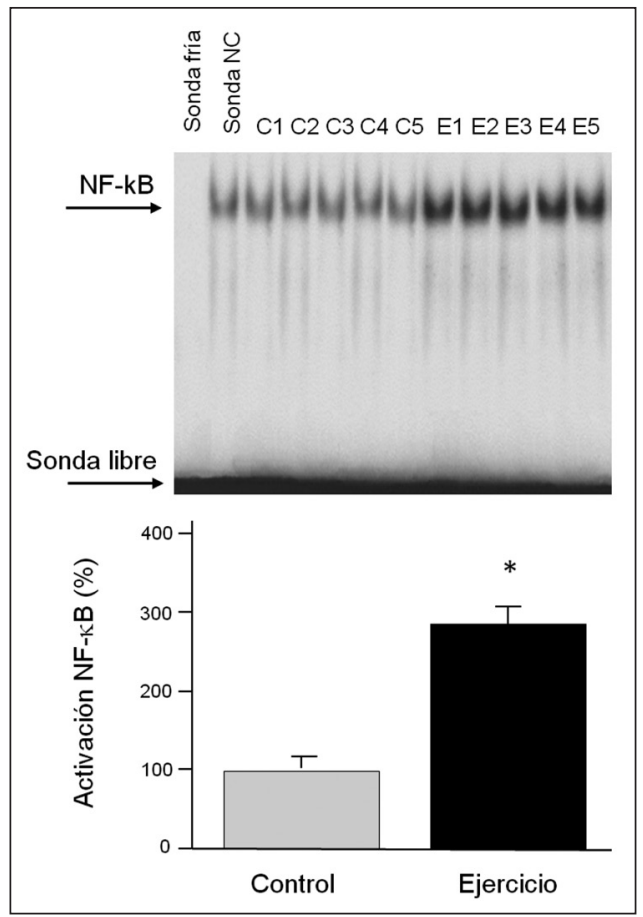

Figura 2. Activación de NF-kB medida a través de la técnica EMSA en tejido cardiaco de ratas sometidas a un protocolo de ejercicio físico agudo. C: Control, E: Ejercicio. Valores medios \pm E.E.M. $(n=16) .{ }^{*} p=0,008$ respecto a control.

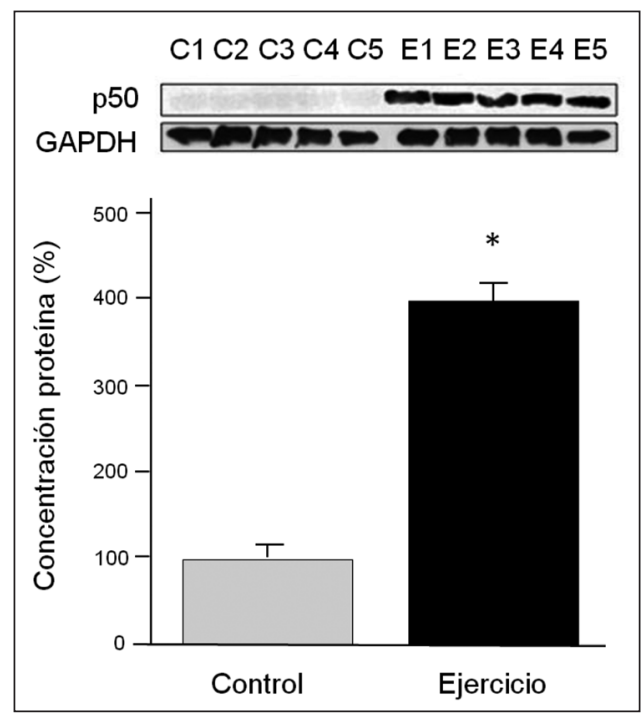

Figura 3. Expresión nuclear de p50 medida a través de la técnica Western blot en tejido cardiaco de ratas sometidas a un protocolo de ejercicio físico agudo. C: Control, E: Ejercicio. Valores medios \pm E.E.M. $(n=16) .{ }^{*} p=0,001$ respecto a control. 
nor inflamación y una disminución en el tamaño del infarto ${ }^{22}$. Sin embargo, nuestros datos sugieren que, la realización de un esfuerzo agudo en animales sin entrenamiento previo, incrementa significativamente los contenidos de ARNm de TLR-4 en el corazón; obteniéndose una información aún no documentada en la literatura científica. Los resultados obtenidos concuerdan con aquellos que pusieron de manifiesto que, tras una única sesión extenuante de ejercicio aeróbico, se incrementaban significativamente los contenidos de TLR-4 en monocitos humanos ${ }^{23}$. Por el contrario, otros autores han demostrado que un programa de entrenamiento físico previo puede disminuir la expresión de TLR-4 en el cerebro de ratas en respuesta a un daño por isquemia/reperfusión ${ }^{24}$.

Se ha especulado que la activación de TLR-4 podría dar lugar a un incremento de los procesos inflamatorios, lo que podría estar asociado con efectos potencialmente perjudiciales para el normal funcionamiento del corazón. Esta hipótesis podría estar avalada por varios estudios. Primero, la activación de diversas citoquinas pro inflamatorias a través del eje TLR-4 y NF- $\kappa$ $\mathrm{B}$ ha sido demostrada en pacientes con infarto de miocardio, fallo cardiaco y angina inestable ${ }^{25}$. Además, se ha propuesto que los TLRs podrían estar involucrados en la relación entre el estilo de vida sedentario con el desarrollo y progresión de enfermedades como la aterosclerosis, fallo cardiaco, enfermedad coronaria, etc ${ }^{26}$, siendo TLR-4 un componente clave en la respuesta innata en el corazón infartado ${ }^{27}$.

Las vías de señalización que culminan en la activación de NF-kB, incluida la vía del TLR-4, se han relacionado con diversos procesos patológicos en el miocardio, incluyendo el aumento de citoquinas proinflamatorias en el cardiomiocito, daño por isquemia/reperfusión, hipertrofia y apoptosis ${ }^{28}$. Actualmente, se está estudiando el NF- $\kappa \mathrm{B}$ como diana terapéutica a nivel molecular ${ }^{29}$, debido principalmente a que se ha demostrado que mantener un bajo nivel de activación de este factor de transcripción generaría cardioprotección ${ }^{30}$. Por tanto, el ejercicio físico regular y no el ejercicio físico agudo de alta intensidad, como queda demostrado en este estudio, podría ser un medio para lograrlo. TLR-4 podría postularse como una proteína con un importante papel en la activación de dicha vía de señalización en el corazón, al igual que sucede en otros órganos o tejidos. Así, estu- dios previos han puesto de manifiesto que, tras la realización de un ejercicio físico extenuante se evidencia un incremento en los contenidos de citoquinas proinflamatorias en el tejido adiposo de ratas. Este efecto parece estar mediado por la vía de señalización del TLR-4 y conduce a la activación del NF- $\kappa B^{31}$. Otras investigaciones, utilizando un inhibidor específico del TLR-4, el eritoran, concluyeron que la vía TLR4/NF- $\kappa \mathrm{B}$ participa en el daño miocárdico durante un modelo de estrés crónico $^{32}$. Por tanto, se debe recalcar que, una carga de trabajo físico agudo podría ser altamente perjudicial para quienes no estén adaptados a este tipo de esfuerzo o quienes posean alguna afección cardiaca. No obstante, en el presente estudio, el papel del TLR-4 sobre la respuesta inflamatoria es especulativo y debería confirmarse con la utilización de inhibidores específicos del TLR-4 o de alguna de las proteínas implicadas en la vía de señalización de dicho receptor.

Por otro lado, los resultados obtenidos sobre la activación del factor de transcripción NF- $\kappa \mathrm{B}$ e iNOS en el tejido cardiaco de ratas son similares a los observados por otros autores ${ }^{11,14}$. Dichos estudios se llevaron a cabo utilizando el mismo protocolo de ejercicio agudo que se ha usado en la presente investigación. El análisis realizado por EMSA reveló una clara activación del NF- $\kappa \mathrm{B}$ en ratas sometidas a ejercicio agudo. Esta mayor traslocación del factor de transcripción fue coincidente con el aumento de la subunidad p50 en núcleos y con la expresión incrementada del ARNm de iNOS. También es importante destacar que está ampliamente demostrado que el incremento de la síntesis de óxido nítrico, produce peroxinitrito, un radical que se ha postulado como uno de los mayores responsables en la muerte celular en este tipo de tejido esquelético ${ }^{33}$. En todas estas investigaciones además se pone de manifiesto que un aumento en los niveles de inflamación incrementa el daño celular en el tejido cardiaco. Por tanto, el ejercicio físico agudo sería responsable de la pérdida de la homeostasis cardiaca, basándose en los cambios observados en los niveles de estrés oxidativo, daño $\mathrm{y}$ apoptosis en cardiomiocitos detectados ${ }^{34}$.

Finalmente, podemos concluir que la mayor expresión de TLR-4 tras el ejercicio agudo parece contribuir a la activación NF- $\kappa \mathrm{B}$, promoviéndose así la transcripción de NOS, lo que podría influenciar negativamente la respuesta cardiaca al esfuerzo físico de alta intensidad. No obstante, es 
indispensable seguir investigando en esta materia y contrastar los datos obtenidos en el presente estudio con la utilización de inhibidores específicos del TLR-4, debiéndose analizar igualmente una variedad más amplia de proteínas mediadoras de la vía de señalización que une TLR-4, NF- $\mathrm{B}$ e inflamación. Además, también sería necesario utilizar diferentes protocolos de ejercicio físico, principalmente programas de entrenamiento, con el fin de profundizar en los efectos del ejercicio físico sobre los mecanismos moleculares implicados en la activación del TLR-4 en este órgano fundamental para la vida.

Agradecimientos: Se agradece a "Beca Presidente de la República de Chile" por el patrocinio brindado al doctorando Carlos Cristi Montero, para la realización de este estudio.

\section{Referencias}

1. Arquer A, Elosua R, Marrugat J. Actividad física y estrés oxidativo. Apunts Med Esport 2010; 45: 31-40.

2. Pedersen BK, Saltin B. Evidence for prescribing exercise as therapy in chronic disease. Scand J Med Sci Sports 2006; 16: 3-63.

3. Löllgen H, Bockenhoff A, Knapp G. Physical activity and all-cause mortality: an updated meta-analysis with different intensity categories. Int J Sports Med 2009; 30: 213-24.

4. Boyd JH, Mathur S, Wang Y, Bateman RM, Walley KR. Toll-like receptor stimulation in cardiomyoctes decreases contractility and initiates an NF-kappaB dependent inflammatory response. Cardiovasc Res 2006; 72: 38493.

5. Trivax JA, Franklin BA, Goldstein JA, Chinnaiyan KM, Gallagher M), deJong AT, et al. Acute cardiac effects of marathon running. J. Appl Physiol 2010; 108: 1148-53.

6. Corrado D, Basso C, Thiene G. Essay. Sudden death in young athletes. Lancet 2005; 366: S47-S48.

7. König D, Neubauer O, Nics L, Kern N, Berg A, Bisse E, et al. Biomarkers of exercise-induced myocardial stress in relation to inflammatory and oxidative stress. Exerc Immunol Rev 2007; 13: 15-36.

8. Gleeson M, McFarlin B, Flynn M. Exercise and Toll-like receptors. Exerc Immunol Rev 2006; 12: 34-53.

9. Takeda K, Kaisho T, Akira S. Toll-like receptors. Annu Rev Immunol 2003; 21: 335-76.

10. Lira FS, Rosa JC, Pimentel GD, Tarini VA, Arida RM, Faloppa F, et al. Inflammation and adipose tissue: effects of progressive load training in rats. Lipids Health Dis 2010; 9: 109-119.

11. Alonso M, Collado PS, González-Gallego J. Melatonin inhibits the expression of the inducible isoform of nitric oxide synthase and nuclear factor kappa B activation in rat skeletal muscle. J Pineal Res 2006; 41: 8-14.

12. Song W, Lu X, Feng Q. Tumor necrosis factor- $\alpha$ induces apoptosis via inducible nitric oxide synthase in neonatal mouse cardiomyocytes. Cardiovasc Res 2000; 45: 595602.

13. World Medical Association, American Physiological Society. Guiding principles for research involving animals and human beings. Am J Physiol Regul Integr Comp Physiol. 2002; 283: R281-R283.

14. Veneroso C, Tuñon MJ, González-Gallego J, Collado PS. Melatonin reduces cardiac inflammatory injury induced by acute exercise. J Pineal Res 2009; 47: 184-91.

15. Jiménez-Jiménez R, Cuevas MJ, Almar M, Lima E, García-López D, De Paz JA, et al. Eccentric training impairs NF-kappaB activation and over-expression of inflammation-related genes induced by acute eccentria exercise in the elderly Mech Ageing Dev 2008; 129: 31321.

16. Sánchez-Campos S, García-Ferreras J, Alvarez M, Jorquera F, Tuñón MJ, González-Gallego J. Rabbit hemorrhagic viral disease: characterization of a new animal model of fulminant hepatic failure J Lab Clin Med 2003; 141: 272-8.

17. Crespo I, García-Mediavilla MV, Gutiérrez MB, Sánchez-Campos S, Tuñón MJ, González-Gallego J. A comparison of the effects of kaempferol and quercetin on cytokine-induced pro-inflammatory status of cultured human endothelial cells. Br J Nutr 2008; 100: 968-76.

18. Lima-Cabello E, Cuevas MJ, Garatachea N, Baldini M, Almar M, González-Gallego J. Eccentric exercise induces nitric oxide synthase expression through nuclean factor-kappaB modulation in rat skeletal muscle. J Appl Physiol 2010; 108: 575-83.

19. Lowry OH, Rosebrough NJ, Farr AL, Randall RJ. Protein measurement with the Folin phenol reagent. J Biol Chem 1951; 193: 265-75.

20. Fillmann H, Kretzmann NA, San-Miguel B, Llesuy S, Marroni N, González-Gallego J, et al. Glutamine inhibits over-expression of pro-inflammatory genes and down-regulates the nuclear factor kappaB pathway in an experimental model of colitis in the rat. Toxicology 2007; 236: 217-26.

21. Crespo I, García-Mediavilla MV, Almar M, González P, Sánchez-Campos S, Tuñón MJ, et al. Differential effects of dietary flavonoids on reactive oxygen and nitrogen 
species generation and antioxidant enzymes in Chang Liver cells. Food Chem Toxicol 2008; 46: 1555-69.

22. Phaneuf S, Leeuwenburgh C. Apoptosis and exercise. Med Sci Sports Exerc 2001; 33: 393-6.

23. Booth S, Florida-James GD, McFarlin BK, Spielmann G. O'Connor DP. Simpson R. The impact of acute strenuous exercise on TLR2, TLR4 and HLA. DR expression on human blood monocytes induced by autologous serum. Eur J Appl Physiol. 2010; 110: 1259-68.

24. Zwagerman N, Plumlee $\mathrm{O}$, Guthikonda M, Ding Y Toll-like receptor-4 and cytokine cascade in stroke after exercise. Neurol Res 2010; 32: 123-6.

25. Hall G, Hasday JD, Rogers TB. Regulating the regulator: NF-kappaB signaling in heart. J Mol Cell Cardiol 2006; 41: 580-91.

26. Pacher P, Beckman JS, Liaudet L. Nitric oxide and peroxynitrite in health and disease. Physiol Rev 2007; 87: 315-424.

27. Birks EJ, Felkin LE, Banner NR, Khaghani A, Barton PJ, Yacoub $\mathrm{MH}$. Increased toll-like receptor 4 in the myocardium of patients requiring left ventricular assist devices. J Heart Lung Transplant 2004; 23: 228-35.

28. Oyama J, Blais C Jr, Liu X, Pu M, Kobzik L, Kelly RA, et al. Reduced myocardial ischemia-reperfusion injury in toll-like receptor 4-deficient mice. Circulation 2004; 109: 784-9.

29. Li H, Malhotra S, Kumar A. Nuclear factor-kappa B signaling in skeletal muscle atrophy. J Mol Med 2008; 86: 1113-26.

30. Gordon JW, Shaw JA, Kirshenbaum LA. Multiple Facets of NF-kappa B in the Heart: To Be or Not to NF-kappa B. Circ Res 2011; 108: 1122-32.

31. Rosa JC, Lira FS, Eguchi R, Pimentel GD, Venancio DP, Cunha CA, et al. Exhaustive exercise increases inflammatory response via Toll like receptor-4 and NF-kBp65 pathway in rat adipose tissue. J Cell Physiol 2011; 226: 1604-7.

32. Wang RP, Yao Q, Xiao YB, Zhu SB, Yang L, Feng JM, et al. Toll-like receptor 4/nuclear factor -kappa B pathway is involved in myocardial injury in a rat chronic stress model. Stress 2011; 14: 567-75.

33. Frantz S, Tillmanns J, Kuhlencordt PJ, Schmidt I, Adamek A, Dienesch C, et al. Tissue-specific effects of the nuclear factor kappaB subunit p50 on myocardial ischemia-reperfusion injury. Am J Pathol 2007; 171: 507-12.

34. Frangogiannis NG. The immune system and cardiac repair. Pharmacol Res 2008; 58: 88-111. 